Thélème. Revista Complutense de Estudios Franceses

ISSN-e: 1989-8193

http://dx.doi.org/10.5209/THEL.54572

\title{
La canción francófona, una herramienta eficaz en el proceso de enseñanza-aprendizaje de la lengua francesa
}

\author{
Maha Mamdouh ${ }^{1}$
}

Recibido: 29 de noviembre de 2016 / Aceptado: 27 de marzo de 2017

Resumen. Este articulo explica los resultados de un estudio que está basado en el uso de la canción francófona en la clase de francés lengua extranjera con el alumnado de secundaria para mejorar la competencia de comprensión auditiva e introducir la cultura francesa mediante un instrumento vivo, real y motivador. Para ello, usamos un corpus de canciones francófonas, con fichas pedagógicas elaborado y adaptado específicamente al aprendizaje de los contenidos y destrezas que deben adquirir los aprendices en este curso. La investigación consiste al principio en determinar el nivel del alumnado antes de la intervención con un pre-test y luego, detectar el cambio producido en esta competencia tras la intervención con un post-test. Además se le pasó un cuestionario al alumnado para que expresase su opinión sobre este método. Al final comparamos este cambio producido en los resultados con los resultados de otro grupo no sometido al tratamiento. Por otro lado, presentamos una muestra del trabajo realizado durante las sesiones de la investigación mediante la canción "C'est de l'eau" del grupo "Les Enfantastiques".

Palabras clave: FLE; canción francófona; enseñanza secundaria; motivación.

\section{[fr] La chanson francophone, un outil efficace dans le processus d'enseignement-apprentissage de la langue française}

Résumé. Cet article explique les résultats d'une étude basée sur l'utilisation de la chanson française en cours de français langue étrangère aux étudiants de l'enseignement secondaire pour améliorer les compétences de compréhension orale et introduire la culture française à travers un outil vivant, réel et motivant. Pour cette fin, nous utilisons un corpus de chansons avec des fiches pédagogiques adaptées spécialement au contenu et aux compétences d'apprentissage à acquérir par les étudiants. En premier lieu, nous avons déterminé le niveau des étudiants avec un pré-test, puis nous avons détecté le changement qui s'est produit après l'intervention avec en plus un post-test et un questionnaire détaillé adressé aux étudiants. En deuxième lieu, nous avons comparé ce changement dans les résultats de nos étudiants avec les résultats d'un autre groupe non soumis au traitement. D'autre part, nous montrons un échantillon du travail effectué au cours des sessions de recherche représenté dans la chanson « C'est de l'eau » interprété par le groupe « Les Enfantastiques ».

Mots clés : FLE; chanson francophone; enseignement secondaire; motivation. 


\title{
[en] Francophone Songs as Efficient Tools in the Process of Teaching and Learning French
}

\begin{abstract}
This article results from a study that uses French songs in the teaching of French as a foreign language at a high school level. The aim is to improve listening skills and to introduce French culture by using in the classroom a real and motivating tool. In order to achieve this goal, we have used a corpus of French songs in tandem with worksheets specifically adapted to the content and skills that students are expected to acquire during the course. In this research first we determine the student's level by means of a pre-test. Then, by giving the students a post-test, we detect possible changes or improvements occurred after applying our teaching method. Finally, we compare our students' results with the results of another group of students not submitted to this method. Additionally, this article presents a sample of the didactic work done during the research, and represented in the song "C'est de l'eau" performed by the group "Les Enfantastiques".
\end{abstract}

Keywords: FLE; Francophone songs; secondry education; motivation.

Sumario: 1. La investigación. 2. La canción "C'est de l'eau". 2.1. Ejemplos de las actividades llevadas a cabo. 3. Los resultados de la investigación. 3.1. La distribución de las puntuaciones obtenidas por los dos grupos. 3.2. Contraste de medias. 3.2.1. Contraste de Medias en las Puntuaciones Diferenciales (muestras independientes). 3.2.2. El Tamaño Del Efecto: Diferencia Tipificada. 3.3. Encuesta. 4. Conclusión, 5 . Referencias bibliográficas.

Cómo citar: Mamdouh, M. (2017). "La canción francófona, una herramienta eficaz en el proceso de enseñanza-aprendizaje de la lengua francesa". Thélème. Revista Complutense de Estudios Franceses, Vol. 32, Núm. 2 : 221-238.

\section{La investigación}

La finalidad de esta investigación es mejorar la práctica educativa de la enseñanza del francés como segunda lengua extranjera, particularmente, la capacitación en comprensión oral (auditiva), mediante la utilización de la canción francófona en el aula. Además, mostrar que con las canciones se puede descubrir la realidad multicultural francófona. Otro objetivo es fomentar el interés de los alumnos y alumnas para aprender francés y motivarlos para continuar con el proceso de desarrollo de destrezas.

La definición de comprensión auditiva en el Diccionario de términos clave del Instituto Cervantes es:

La compresión auditiva es una de las destrezas lingüísticas, la que se refiere a la interpretación del discurso oral. En ella intervienen, además del componente estrictamente lingüístico, factores cognitivos, perceptivos, de actitud y sociológicos. Es una capacidad comunicativa que abarca el proceso completo de interpretación del discurso, desde la mera descodificación y comprensión lingüística de la cadena fónica (fonemas, sílabas, palabras, etc.) hasta la interpretación y la valoración personal; de modo que, a pesar de su carácter receptivo, requiere una participación activa del oyente (Centro Virtual Cervantes, 2016). 
También, la competencia de la comprensión oral está incluida entre las actividades comunicativas de la lengua en El Marco europeo de referencia. El desarrollo de esta competencia se puede concretar en la evolución de las siguientes destrezas:

1. captar el objetivo del discurso;

2. discriminar las ideas principales de las complementarias;

3. discriminar las palabras importantes de las secundarias;

4. anticipar la continuación del discurso;

5. inferir aquello que no se explicita, captar las implicaturas;

6. detectar e interpretar el sentido figurado, el doble sentido, los juegos de palabras, la ironía, el humor, en general, las falacias;

7. captar el estado de ánimo y la actitud del emisor (Marco común europeo de referencia para las lenguas, 2001).

En las últimas décadas, los investigadores se han dado cuenta de que la comprensión oral no es un proceso de recepción pasiva de las informaciones, sino un proceso activo de selección, codificación, almacenamiento y reconstitución influida por factores diversos tales como lingüísticos, cognitivos, culturales, sociales, etc.

On commence prendre conscience que la compréhension orale n'est pas un processus de réception passive des informations, mais un processus actif de sélection, encodage, stockage et reconstitution influencée par divers facteurs tels que linguistiques, cognitifs, culturels, sociaux etc. (Guan, 2005: 8).

De modo que buscamos exponer a nuestros alumnos a la comprensión oral del francés en situaciones cotidianas y de la vida real reflejada en las canciones. Partimos del presupuesto de que la canción promueve el conocimiento de rasgos que distinguen la cultura de los territorios en los que se habla la lengua que es objeto de aprendizaje. Según Boiron "[1]a chanson est un lien avec la culture de l'autre dans sa diversité. Elle est un lieu de découverte de la réalité multiculturelle française et francophone. Elle a aussi une mission de plaisir, de divertissement" (Boiron, $1997: 1$ ).

Jean-Louis Calvet (1977: 8), antes que Boiron, enunciaba esta idea: "apprendre une langue c'est apprendre ou tenter de pénétrer une culture, et que la chanson est, entre autres éléments, constitutive de cette culture".

Igualmente, la adquisición de la competencia sociocultural mediante la canción francesa fue ilustrada con la valiosa propuesta de Pierre Dumont, en colaboración con Renaud Dumont, presentada en su libro Le Français par la chanson (1998). Este desarrolla un estudio de los antecedentes metodológicos y sociológicos en la enseñanza de la cultura francesa en la clase de francés.

Aunque las canciones no son creadas, en principio, con fines pedagógicos, se consideran uno de los documentos auténticos por excelencia que se pueden trabajar en la clase de la lengua extranjera. Por un lado, ofrecen la oportunidad a los estudiantes de trabajar la lengua actual usada en la vida cotidiana, por otro lado, les permiten acercarse a la cultura francófona.

Existen muchos argumentos a favor del uso de la canción en la clase de FLE, pero los más interesantes, a nuestro parecer, son los que ofrece Louis-Jean Calvet en forma de definición en su libro La chanson en classe de français langue étrangère (1980: 8): 
C'est un micro-univers signifiant dont l'organisation textuelle est close et fortement structurée. C'est un genre spécifique dans lequel les éléments linguistiques et extralinguistiques sont étroitement liés et se combinent. Donc, c'est un mélange de linguistique, de mélodique et de rythmique. [...] C'est le lieu d'un sens composé, le lieu d'une convergence entre procédés mélodiques et procédés linguistiques.

Más lejos (1980: 20), Calvet añade: "Elle est langue, elle est culture, elle est plaisir et elle peut être moyen pédagogique".

Evidentemente, en el presente estudio, la palabra canción hace referencia a una composición de letras cantadas, individualmente o colectivamente, que suelen ir acompañadas de música, tiene el objetivo de proporcionar una oportunidad para que los estudiantes puedan tener una comprensión correcta de la lengua francesa. La tomamos también como una herramienta que sirve para ayudar a los estudiantes a escuchar, a distinguir los sonidos de las palabras, que pueden ser repetidos sin que resulte excesivamente monótono para ellos $\mathrm{y}$, como consecuencia, puede permitir mejorar la comprensión oral de los estudiantes de secundaria, así como incrementar su gusto y su interés por aprender la lengua francesa.

Así pues, en nuestro campo de la enseñanza de las lenguas extranjeras podemos identificar la canción, por lo menos, con las tres características siguientes:

1. Se trata de un documento auténtico, es decir, que no se ha producido con el fin de estudiarlo en la clase de FLE.

2. Se trata de un documento sonoro, es decir, un documento que se puede trabajar en la clase para mejorar la comprensión oral.

3. Se trata de un documento cultural, es decir, que pertenece a la vida cotidiaa de la sociedad francófona, que expresa su realidad, sus noticias, sus ideologías, determinados comportamientos y usos, etc.

En cualquier caso, el uso de la canción en la enseñanza de la lengua francesa no es algo nuevo. La historia moderna de la utilización de la canción francófona en la clase de francés lengua extranjera empezó a finales de los años 50, cuando el manual Cours de langue et de civilisation française (Mauger, 1955) recogió ocho canciones populares acompañadas de las correspondientes fichas pedagógicas como, por ejemplo, Alouette y Auprès de ma blonde. Después, otros métodos siguieron este ejemplo en el mismo sentido, la utilización didáctica de canciones populares.

Las metodologías dominantes en ese momento, es decir, la metodología audio-oral y la metodología tradicional, basada en la gramática y la traducción contaban de este modo con actividades excelentes de memorización y de automatización de las estructuras de la lengua, gracias a la repetición de estribillo de la canción. Además, mantenían los ejercicios con estructuras gramaticales a partir de ellas. Por otro lado, los estudiantes se solían ejercitar en el canto coral para respetar los aspectos musicales.

En los años 60 algunos profesores pasaron de las canciones populares tradicionales a las canciones modernas. Al final de dicha década, se inició la polémica en torno a cómo determinar los criterios de selección de la canción adecuada. Desafortunadamente, las letras de las canciones fueron el único criterio que se tuvo en cuenta para valorar una canción. Estaban interesados solo en el texto, sin considerar en absoluto 
el resto de componentes, como su riqueza musical o el contexto en el que se había creado. Además, en gran medida, se seguía sin atender a los gustos de los jóvenes o los adolescentes.

Gracias a los escritos de Louis-Jean Calvet, especialmente "Chanson: Quelle stratégie?" (1979), todos los tipos de música, incluso los que poco antes eran vistos como comerciales, inaudibles, arrastrados o vulgares, pues contenían palabras y expresiones argóticas comenzaron a ser utilizados en el aula.

En 1992, surge la iniciativa de Michel Boiron y Patrice Hourbette de dar a conocer la nueva producción de música francesa por todo el mundo. Publicaron para ello una serie de CDs llamados Generación francés, en la que cada disco contiene una colección de canciones, acompañadas de sus guías didácticas y de las biografías de los artistas para el aprovechamiento pedagógico de cada tema musical y un mejor uso de la canción en la clase de FLE. Uno de los primeros objetivos de estos autores era sensibilizar a los profesores de FLE sobre la utilidad de esta herramienta en el aula y, por supuesto, ayudarlos a descubrir nuevas tendencias en la música francesa.

De acuerdo con la idea de la conciencia intercultural, Boiron señaló la importancia de exponer a los estudiantes a la cultura francófona y no únicamente a la cultura francesa. Ello se produce, entre otras cosas, mediante la elección de la canción francófona en general para la clase de FLE. En su artículo "Rencontrer la musique française d'aujourd'hui" (1998), este autor afirma que la canción contemporánea refuerza la actualización de la lengua aprendida y la inserción en el mundo actual.

Esta idea la encontramos claramente por primera vez en el cuarto disco de la colección a la que nos hemos referido, Nouvelle génération française 4, (2002). Este aportó alguna novedad en comparación con los anteriores, entre otras, recogía no solo canciones francesas sino también canciones cuyos compositores o intérpretes eran originarios de otros países de habla francesa. De hecho, en ese CD se pueden encontrar canciones de artistas francófonos como Amadou et Mariam de Mali, Samia Farah de Túnez, Tété de Senegal, así como los grupos musicales, Bisso Na Bisso franco-congoleño y Sawt El Atlas de Marruecos.

Por otro lado, el centro pedagógico CAVILAM (Centre d'Approches Vivantes des Langues et des Médias), en la ciudad de Vichy, del que Boiron es el actual director, acoge cada año a decenas de profesores de francés de diferentes nacionalidades para que aprendan y desarrollen aún más sus habilidades para enseñar la lengua y la civilización francesas mediante el uso de este instrumento cultural.

Desgraciadamente, a pesar de estas iniciativas promocionales del uso de la canción en la enseñanza de la lengua y el reconocimiento general de su utilidad, la canción sigue estando muy poco presente en los manuales de FLE.

En nuestro caso, nos hemos inspirado en todas las investigaciones anteriores para elaborar nuestro estudio y aprovechamos sus experiencias, intentando sacar el máximo partido para llegar a proponer unas prácticas que sean verdaderamente útiles en nuestras clases y en nuestra investigación.

Nuestro experimento trata de realizar actividades con canciones durante diez sesiones, duración del proyecto. Nos referimos a la incorporación de las estrategias de enseñanza de comprensión oral con actividades a partir de canciones francófonas.

Nos propusimos utilizar un corpus de canciones que pudiera interesar al alumnado de Enseñanza Secundaria y que se adaptase al aprendizaje de los contenidos y destrezas que deben adquirir. Para ello, elaboramos una guía pedagógica con los 
materiales y fichas pedagógicas que consideramos necesarios para conseguir la finalidad que deseamos.

El sitio Web TV5MONDE nos resultó de gran ayuda en la elaboración de nuestra guía pedagógica. Asimismo, nos pareció muy interesante y adecuado para ayudar a los profesores de abordar este tipo de actividades. El sitio ofrece una gama de temas, recursos, herramientas y servicios gratuitos para enseñar y aprender la lengua francesa. Nos interesaba, sobre todo, sus materiales de comprensión oral que las proporciona mediante un conjunto de herramientas para explotar las emisiones del canal, tal como los informes de reportajes y las canciones más modernas.

Durante dicho trabajo explotamos con nuestros alumnos y alumnas las siguientes canciones:

1. Être un homme comme vous : Ben l'Oncle Soul.

2. C'est de l'eau : Les Enfantastiques.

3. Laissons-les rêver : John Mamann.

4. Soleil soleil : Lara Fabian.

5. Le même sang : Yannick Noah

6. Mon loup: Laura Cahen

7. Ouvre moi la porte: Enrico Macias

8. On écrit sur les murs: Kids United.

9. Manger un croissant: Carlos

10. J'ai peur du noir: Aldebert, Elodie Frégé y Vincent Baguian.

Igualmente, proponemos llevar al aula todo tipo de canciones que pueden destacar la riqueza y la diversidad de la creación contemporánea en Francia y en todo el mundo francófono, dado que, como ya hemos señalado, la elección de las canciones más recientes actualiza, refuerza el lenguaje aprendido y favorece la inserción en el mundo de hoy.

En la elección de dichas canciones, seguimos los siguientes criterios, inspirados por Boiron en su artículo "Approches pédagogiques de la chanson" (1997: 1):

- Que sea apropiada para la utilización en la clase, según el profesor.

- Que corresponda a los hábitos de escucha de los estudiantes o esté de moda.

- Que sorprenda o que sea atípica.

- Que el tema de la canción corresponda al tema abordado en clase.

- Que sea posible cantarla, sea bailable o se pueda utilizar para organizar un espectáculo.

- Que sea propuesta por alguno de los estudiantes.

- Que se escuche en la radio o sea un éxito de ventas.

La investigación tuvo lugar en un colegio privado en Benalmádena (Málaga) en el que toda la enseñanza se imparte en inglés. El ensayo se hizo con dos grupos de estudiantes de entre 12 y 14 años. Para poder evaluar la práctica trabajamos con el grupo natural de la clase de "year 8" del centro, como grupo experimental, y los estudiantes del curso posterior como grupo de contraste, es un grupo que es un año mayor y, por lo tanto, al comienzo del experimento llevaban más tiempo estudiando francés siguiendo un método más tradicional. Son dos clases impartidas por la misma profesora. 
El estudio está basado en un diseño cuasi-experimental, el más utilizado en el ámbito educativo. Según este diseño, tenemos un grupo experimental, sometido al tratamiento, y un grupo de contraste no sometido al tratamiento, con pre y post-test en los dos grupos.

La intervención consiste en una hora semanal, durante diez semanas. En cada sesión, hacemos varias escuchas de la misma canción. La primera escucha de la canción se considera como el ejercicio más simple en el que los estudiantes tratan de averiguar el sentido general de ella, incluso cuando su letra no se haya comprendido en su totalidad. Tras la primera escucha, intercambiamos algunas observaciones sobre el cantante y el tema de la canción como; ¿Quién canta? ¿Es famoso? ¿Es joven o de mediana edad? ¿Cómo les parece el tono? ¿Triste, alegre, melancólico, agresivo, violento, nostálgico o irónico?, ¿A quién se dirige la canción?, ¿Quiénes son las protagonistas del vídeo? Este conjunto de preguntas puede ir más allá, de acuerdo con la comprensión en la primera escucha. Escuchar y comentar puede proporcionar acceso a la comprensión de la canción, lo que es un paso enorme.

A continuación se les da la letra, se vuelve a escuchar la canción otra vez, y mientras los estudiantes están haciendo las actividades se vuelve a escuchar la canción una o varias veces según sea necesario. En cada sesión se trabajan algunas actividades relacionadas con el desarrollo de la destreza de la comprensión auditiva que son, por ejemplo, responder a preguntas sobre el contenido del texto oído, colocar versos o palabras previamente desordenadas etcétera, también se repasan las estructuras y el vocabulario previstos (que son objeto de la sesión).

Después de haber escuchado la canción varias veces, aprenden la música y por último pueden cantarla. Se canta la canción con el grupo clase, incluso, muchas veces algunos alumnos o alumnas se atreven a cantar solos. Y así se producen cadenas de palabras completamente correctas, algo que mejora la pronunciación.

\section{La canción "C'est de l'eau"}

Enseñar un idioma es enseñar la lengua, pero también la cultura del pueblo o de los pueblos que lo hablan y a veces hablar del universo.

Escogimos la canción "C'est de l'eau", como ejemplo del trabajo realizado. Esta fue una de las canciones trabajadas con los estudiantes durante las sesiones de mi investigación. El agua, desde diferentes perspectivas, geográfica, económica, lúdica e incluso sanitaria o de bienestar, representaba un tema esencial en el contenido del programa del grupo experimental. Así que quisimos abordar este tema mediante la canción "C'est de l'eau" del grupo "Les Enfantastiques", una canción sencilla y a la vez con considerable vocabulario correspondiente al tema conforme al nivel de nuestros estudiantes. Gracias a las actividades elaboradas para dicha canción pudimos practicar el vocabulario relacionado con este tema y trabajar diferentes objetivos como:

- Objetivos comunicativos.

- Hablar de la importancia del agua para la salud y nuestro organismo. 
- Hablar de las actividades relacionadas con el agua.

- Hablar de la representación del agua en el planeta y en nuestro cuerpo.

- Objetivo cultural:

- La hidrografía de Francia y sus principales ríos y mares.

- Objetivo intercultural:

- La diferencia de la disponibilidad del agua en las diferentes zonas del mundo.

- La conciencia mundial con respecto al consumo responsable del agua.

\subsection{Ejemplos de las actividades llevadas a cabo}

Empezamos la clase escuchando la canción. Luego, hacemos una exposición del tema y suscitamos la discusión para fomentar la interacción oral, preguntando a los alumnos sobre las actividades lúdicas y vitales que pueden estar relacionadas con el agua. Los alumnos mencionaron numerosas actividades lúdicas y deportivas tales como nadar o bucear. Así como actividades cotidianas relacionadas con el tema (ducharse, lavarse), y preguntaron varias veces sobre el modo de expresar algunas actividades en francés.

También dimos a conocer el grupo "Les Enfantastique" y su creador "Monsieur Nô", Se trata de un coro formado por alumnos reales que ensayan y graban las canciones en su colegio. Sus canciones son conocidas en numerosos países. Este coro les gustó mucho y les hizo mucha ilusión saber que niños como ellos cantaban y eran conocidos fuera de su país a mis alumnos y alumnas.

Tras escuchar la canción dos veces y explicar el significado del nuevo vocabulario, empezamos las actividades siguientes:

\section{Actividad (1)}

Observez la carte ci-dessus et écrivez les noms des principaux fleuves et des principales rivières de France.

$1-$
$2-$
$3-$
$4-$

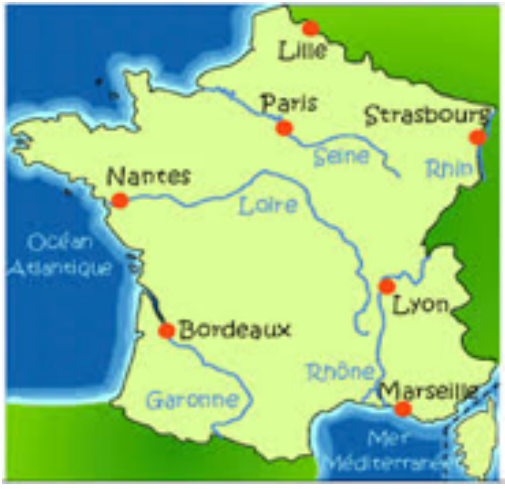

Fig. 1 Fleuves de France. maxicours.com 
- Estructura: Se trata de un mapa Hidrográfico de Francia con sus principales ciudades, en el que los estudiantes debían reconocer los nombres de los ríos.

- Modalidad de trabajo: individual o en grupos de dos.

- Mostrar el mapa a los estudiantes.

- Explicar la diferencia entre "fleuve" y "rivière" y las características de cada uno de ellos.

- Relacionar los ríos con las ciudades que atraviesan.

- Hablar de la dirección de los cauces, norte, sur, este, oeste.

- Pedir a los alumnos que interpreten el mapa y que escriban los nombres de los ríos junto a los números.

- Hacer un comentario colectivo sobre las ciudades que aparecen en el mapa.

- Objetivo: Se trata de conocer los principales ríos de Francia así como sus principales ciudades y situarlos en el mapa.

- Competencias trabajadas:

- Competencia matemática y competencias básicas en ciencia y tecnología: Consiste en aplicar el razonamiento matemático y sus herramientas para describir, interpretar y predecir distintos fenómenos en el contexto del mapa y de distinguir entre "fleuve" y "rivière" según el concepto francés.

- Competencia de autonomía e iniciativa: Los estudiantes verifican sus hipótesis a la hora de distinguir entre los dos tipos de ríos según el concepto francés.

- Competencia social y ciudadana: Comprender y analizar de manera crítica los usos generalmente aceptados en las distintas sociedades y entornos.

- Aprender a aprender: Los alumnos y alumnas deben ser capaces de apoyarse en experiencias vitales y de aprendizaje previas, como la geografía, con el fin de utilizar y aplicar los nuevos conocimientos y capacidades en otros contextos, como el aprendizaje de la lengua extranjera.

- Observaciones: Los alumnos mostraron su extrañeza ante la diferencia entre el concepto español y el concepto francés al definir los ríos. También mostraron mucho interés en los nombres de las ciudades francesas. Algunos estudiantes comentaron que habían visitado algunas de las ciudades mencionadas. 
Actividad (2)

Reliez les expressions avec les éléments de droite.

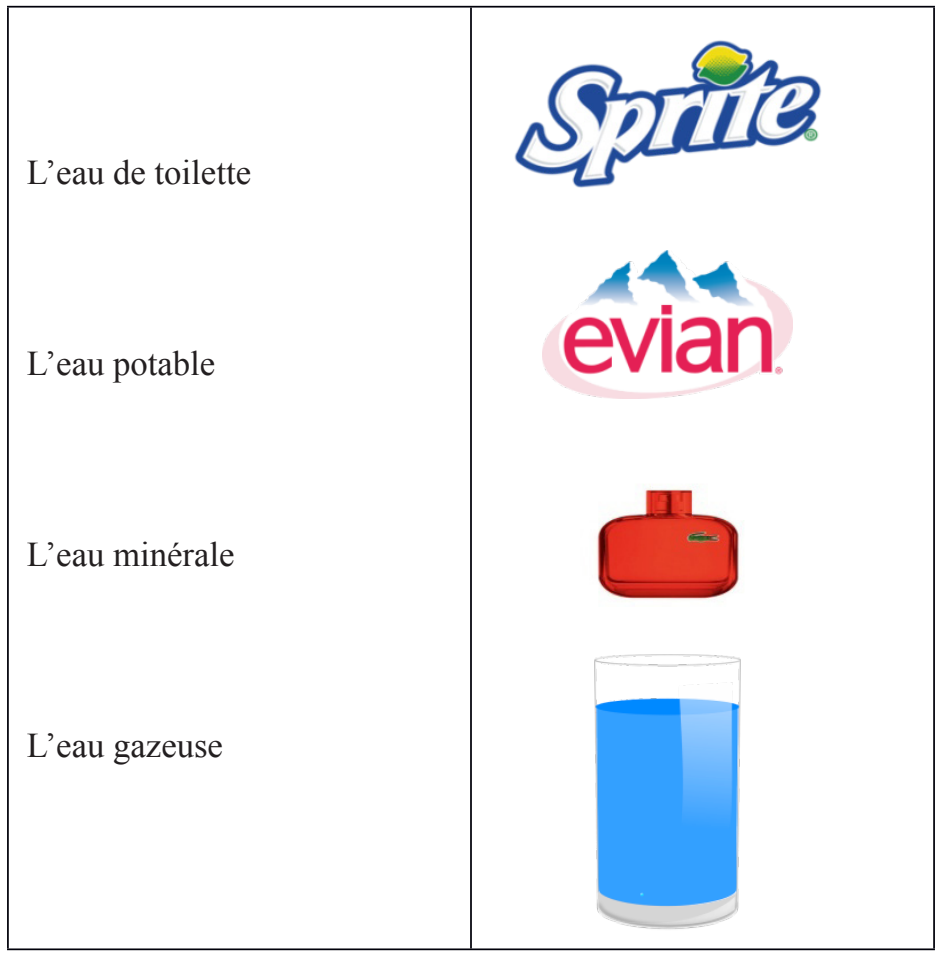

- Estructura: Se trata de diferentes expresiones relacionadas con vocabulario del tema del agua. Los estudiantes deben unir cada expresión con la imagen correspondiente.

- Objetivo: Trabajar y enriquecer el vocabulario correspondiente al tema.

- Modalidad de trabajo: en grupos de 2.

Explicar a los alumnos el significado de cada expresión para que puedan reconocer la imagen correspondiente.

- Competencias:

- La autonomía e iniciativa para encontrar la imagen clave de cada expresión.

- Observaciones: El vocabulario de esta actividad es nuevo para el alumnado por lo que necesitaba una explicación previa. Los alumnos y alumnas lo hicieron con ilusión porque algunas palabras les suenan de la publicidad tal como la palabra "eau de toilette". 
Actividad (3)

Dites si c'est « vrai » ou « faux ».

a) On surnomme la terre "la planète bleue" car vue de l'espace, notre terre est bleue.

b) 1 faut boire de l'eau régulièrement.

c) Boire beaucoup d'eau n'est pas bon pour la santé.

d) $70 \%$ de la surface de la Terre est recouverte d'eau.

- Estructura: Tratar de contestar con "verdadero" o "falso" sobre cuatro frases que representan datos del agua.

- Objetivo: Ir más allá de la lengua y conocer algunos datos y costumbres saludables relacionados con el agua.

- Modalidad de trabajo: Individual o en grupo de dos.

- Competencias:

- Competencia lógica: Ejercer la lógica para asociar lo verdadero y lo falso.

- Competencia social y ciudadana: interesarse sobre el conocimiento del medio.

- Aprender a aprender: Los estudiantes deben ser capaces de apoyarse en experiencias vitales y de aprendizaje previas con el fin de utilizar y aplicar los nuevos conocimientos y capacidades en otros contextos, como la de la naturaleza en la lengua extranjera.

Actividad (4)

Cochez et ordonnez les mots entendus dans le clip

rivières nuages neige fleurs nature bateau

océans lune soleil une rivière ruisseaux pluie

- Estructura: Se escucha la canción de nuevo, con el fin de determinar la existencia de dichas palabras en la letra de la canción. Una vez que se ha determinado si las palabras mostradas en la ficha se hallaban en la canción se pasaba a ponerlas en orden según su aparición en el desarrollo del tema musical.

- Objetivo: desarrollar la comprensión oral y aprender a distinguir los sonidos y las palabras oídos.

- Modalidad de trabajo: individual.

- Competencias:

- La competencia lógica: utilizar la lógica y analizar el texto oído para diferenciar entre las palabras.

- Observaciones: Los alumnos y alumnas pidieron escuchar la canción más veces para asegurarse de sus respuestas y escuchar las palabras que les faltaban. Algunos estudiantes mostraron algunas dificultades para encontrar algunas palabras 
Al final de la sesión pedimos a los alumnos que buscasen otras canciones interpretadas por el grupo "Les Enfantastiques" para reforzar su interés por aprender.

\section{Los resultados de la investigación}

En el análisis de los resultados obtenidos después de incorporar el tratamiento, se ha notado un cambio positivo en el grupo experimental, en la destreza de la comprensión oral trabajada durante las sesiones del experimento y examinada con el pre y post-test. Los resultados obtenidos y presentados aquí demuestran que hubo diferencias entre los dos grupos después del tratamiento. Aunque el grupo de contraste dominó los promedios mayores en el pre-test, los promedios mayores en el post-test fueron obtenidos por el grupo experimental.

\section{Tabla 1. RESULTADOS DE LAS PUNTUACIONES DEL PRE-TEST}

\begin{tabular}{lcc}
\hline & $\begin{array}{c}\text { Grupo } \\
\text { experimental }\end{array}$ & $\begin{array}{c}\text { Grupo de } \\
\text { contraste }\end{array}$ \\
\hline Promedio & 35.73 & 39.88 \\
Desviación Estándar & 3.85 & 6.38 \\
\hline
\end{tabular}

En la tabla 1 se presentan los promedios de las puntuaciones del pre-test. E1 grupo experimental obtuvo un promedio de 35.73 con una desviación estándar de 3.85 . Mientras que e1 grupo contraste obtuvo un promedio de 39.88 con una desviación estándar de 6.38 .

Tabla 2. RESULTADOS DE LAS PUNTUACIONES DEL POST-TEST

\begin{tabular}{lcc}
\hline & $\begin{array}{c}\text { Grupo } \\
\text { experimental }\end{array}$ & $\begin{array}{c}\text { Grupo de } \\
\text { contraste }\end{array}$ \\
\hline Promedio & 41.73 & 38.94 \\
\hline Desviación Estándar & 6.08 & 4.21 \\
\hline
\end{tabular}

De los resultados de los promedios de las puntuaciones presentadas del post-test en la tabla 2, se desprende que el grupo experimental fue el que obtuvo el promedio mayor (41.68), seguido del grupo control con un promedio más bajo 38.94.

\subsection{La distribución de las puntuaciones obtenidas por los dos grupos.}

En la tabla 3, se puede observar la distribución de las puntuaciones obtenidas por los estudiantes en el pre-test. Se incluye la distribución de las puntuaciones obtenidas en los dos grupos, utilizando la escala 1-18, 19-28, 29-38, 39-48, y la frecuencia de estudiantes que se encuentran en estos intervalos, la finalidad de esta distribución es observar tendencias, así como describir el porcentaje de estudiantes que dominó las destrezas en la pre-test y en el post-test del grupo experimental y el grupo de con- 
traste. El ochenta y dos por ciento (82\%) de los estudiantes del grupo experimental se ubicó en el intervalo de las puntuaciones 2938 y el dieciocho por ciento $(18 \%)$ obtuvo puntuación entre 39-48. Mientras que el sesenta y tres por ciento $(63 \%)$ de los estudiantes del grupo contraste se ubicó en el intervalo de dichas puntuaciones $39-48$, el veinticinco por ciento (25\%) obtuvo puntuaciones entre $29-38$ y solo el trece por ciento (13\%) obtuvo puntuaciones entre 19-28.

\section{Tabla 3. DISTRIBUCIÓN DE LAS PUNTUACIONES OBTENIDOS POR LOS ESTUDIANES EN EL PRE-TEST.}

\begin{tabular}{lllll}
\hline \multirow{2}{*}{ Puntuación } & \multicolumn{2}{c}{$\begin{array}{c}\text { Grupo experimental } \\
\text { N=11 }\end{array}$} & \multicolumn{2}{c}{$\begin{array}{c}\text { Grupo de contraste } \\
\text { N=8 8 }\end{array}$} \\
\cline { 2 - 5 } & Frecuencia & $\%$ & Frecuencia & $\%$ \\
\hline $1-18$ & 0 & $0 \%$ & 0 & $0 \%$ \\
\hline $19-28$ & 0 & $0 \%$ & 1 & $13 \%$ \\
\hline $29-38$ & 9 & $82 \%$ & 2 & $25 \%$ \\
\hline $39-48$ & 2 & $18 \%$ & 5 & $63 \%$ \\
\hline
\end{tabular}

En la tabla 4 se presenta la distribución de las puntuaciones obtenidas por los estudiantes de los dos grupos en el posttest, que es el resultado obtenido después de incorporar el tratamiento en el grupo experimental, se incorporaron las estrategias de enseñanza de comprensión oral con actividades de canciones francófonas, mientras que en el grupo control no se incorporó dicho tratamiento.

\section{Tabla 4. DISTRIBUCIÓN DE LAS PUNTUACIONES OBTENIDOS POR LOS ESTUDIANES EN EL POST-TEST.}

\begin{tabular}{lllll}
\hline \multirow{2}{*}{ Puntuación } & \multicolumn{2}{c}{$\begin{array}{c}\text { Grupo de Control } \\
\text { N= 11 }\end{array}$} & \multicolumn{2}{c}{$\begin{array}{c}\text { Grupo de contraste } \\
\text { N=8 }\end{array}$} \\
\cline { 2 - 5 } & Frecuencia & $\%$ & Frecuencia & $\%$ \\
\hline $1-18$ & 0 & $0 \%$ & 0 & $0 \%$ \\
\hline $19-28$ & 0 & $0 \%$ & 0 & $0 \%$ \\
\hline $29-38$ & 2 & $12 \%$ & 2 & $25 \%$ \\
\hline $39-48$ & 9 & $82 \%$ & 6 & $75 \%$ \\
\hline
\end{tabular}

En la distribución de las puntuaciones del post-test (tabla 4), se observa que el porcentaje mayor de estudiantes ubicados entre las puntuaciones 39-48 eran estudiantes del grupo experimental (82\%). Mientras el porcentaje de estudiantes ubicados en este intervalo de puntuaciones del grupo de contraste representan el $75 \%$.

A pesar de que este último grupo comenzó con un aprovechamiento más alto en el pre-test con un promedio de 39.88 es decir, una diferencia de 4,15 puntos más alto que el grupo experimental, que obtuvo un promedio de solo 35.73, aquel finalizó con un aprovechamiento más bajo en la comprensión oral en ambos promedio con una diferencia de 2.74 con respecto al promedio del grupo experimental y también por 
lo que se refiere al porcentaje de los estudiantes cuyas puntuaciones se situaron en el intervalo más alto.

\subsection{Contraste de medias}

\subsubsection{Contraste de Medias en las Puntuaciones Diferenciales (muestras independientes)}

En el análisis de contraste de medias, tanto del grupo experimental como del grupo de contraste se calcula para cada grupo una puntuación de cambio o puntuación diferencial (post-test - pre-test)

Tabla 5. Puntuación diferencial entre el post-test - pre-test

\begin{tabular}{lllllll}
\hline & \multicolumn{2}{c}{ Grupo experimental, $\mathbf{N}=\mathbf{1 1}$} & \multicolumn{3}{c}{ Grupo de contraste, $\mathbf{N}=\mathbf{8}$} \\
\hline & Pre-test & Post-test & Post menos pre & Pre-test & Post-test & Post menos pre \\
\hline media & 35.73 & 41.73 & $\mathbf{6}$ & 39.88 & 38.94 & $\mathbf{- 0 . 9 4}$ \\
\hline \multirow{2}{*}{ desviación } & 3.85 & 6.08 & & 6.38 & 4.21 & \\
\hline
\end{tabular}

Colocamos estos valores en la tabla 5 donde se nota la diferencia entre las medias, lo que se considera un dato importante. Vemos que la puntuación diferencial del grupo experimental es positiva con una cifra significativa de "6 puntos" mientras que la puntuación diferencial del grupo de contraste es negativa en "-0.94 puntos".

\subsubsection{El Tamaño Del Efecto: Diferencia Tipificada}

El tamaño del efecto es una cuantificación de la diferencia, es decir, es la diferencia entre las dos medias dividida por la desviación típica combinada de los dos grupos; el tamaño del efecto más habitual es el de Cohen (1988) simbolizado con la letra "d".

$$
\mathbf{d}=\frac{\text { media mayor-media menor }}{\text { Desviación típica combinada de las dos muestras }}
$$

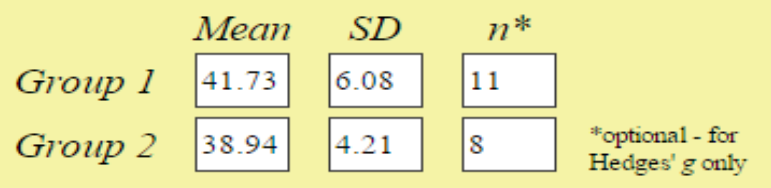

Cohen's $d=0.5335$

Fig. 2 Paul Ellis, The Hong Kong Polytechnic University, Effect Size FAQs 
Los criterios para valorar estos tamaños del efecto son:

$\mathrm{d}=.20$ pequeño

$\mathrm{d}=.50$ moderado

$\mathrm{d}=.80$ grande (Cohen, 1988: 26).

El tamaño del efecto de nuestro estudio ha sido, como mostramos en las imágenes, $\mathbf{0 . 5 3 3 5}$, es decir que está situado en los criterios moderados. "Por diferencias moderadas (en torno a $\mathrm{d}=.50$ ) se entienden aquí aquellas diferencias entre grupos que pueden detectarse por simple observación” (Morales, 2008: 290).

Convencionalmente suele considerarse un valor de $\mathrm{d}=.50$ como de significación práctica (importante); cuando se trata de resultados de rendimiento escolar o de investigación educacional se consideran de relevancia práctica valores en torno a .30 (Morales, 2008: 290; Valentine y Cooper, 2003: 5).

\subsection{Encuesta}

Por último, para medir la motivación del alumnado tras las sesiones así como su valoración sobre lo que han aprendido, se ha realizado una encuesta para responder con sí o no a varias preguntas

Aunque las notas sean un criterio válido para verificar el éxito de una práctica didáctica, no olvidamos otros efectos que puede merecer la pena medir y evaluar, como son posibles cambios en:

- La autoeficacia (percepción de la propia competencia).

- El gusto por la clase, de francés, en este caso.

- La percepción del propio aprendizaje (no simplemente del aprendizaje que se puede medir con un examen), determinadas actitudes o valores, etc. (Morales, 2013: 25).

Por eso, los alumnos del grupo experimental respondieron a un cuestionario para dar sus impresiones, y les preguntamos si esta práctica: Les ha gustado, les ha motivado para ir clase, les ha estimulado intelectualmente para aprender más francés, entre otras preguntas. El resultado ha sido el siguiente:

1. Me gustan las clases sobre la canción francesa.

Los 11 estudiantes de la clase respondieron con sí a esta pregunta. Estaban impacientes, esperaban el día de las clases de francés en que iban a escuchar una nueva canción.

2. Esta clase me anima para intentar aprender más francés.

Un $82 \%$ de los estudiantes, o sea 9 de los 11 respondieron con un sí, que esta clase les anima a aprender más francés y que se sienten más atraídos por el aprendizaje de la lengua. 
3. La clase me motiva a escuchar más canciones y audios franceses.

Sobre si la clase los motiva para escuchar más canciones y audios franceses, concretamente, 9 de los 11 estudiantes respondieron que sí.

4. En esta clase he aprendido la pronunciación correcta de algunas palabras del francés.

Sobre esta pregunta, 10 de los 11 estudiantes respondieron que sí. Con la repetición de las canciones se escuchan más veces las palabras y se aprende su correcta pronunciación.

5. La clase ha mejorado mi comprensión oral del francés.

De nuevo tenemos que un 91\% respondieron afirmativamente. Escuchar cada canción varias veces durante la sesión hace que les quede grabada la pronunciación correcta de cada frase y les resulta más fácil captar el mensaje del audio.

6. Me gustaría que siguiésemos con clases en las que se trabajase con canciones francesas.

A la totalidad de los estudiantes les gustaría seguir aprendiendo mediante este método como se puede observar en el gráfico. Esto demuestra la aceptación de este método por parte de los alumnos y alumnas.

\section{Conclusión}

La canción es una herramienta que se adapta a diversos aspectos de la enseñanza de las lenguas extranjeras. Contiene todos los componentes lingüísticos, semánticos y culturales que necesitan los docentes en su enseñanza, además de un factor motivador para los estudiantes. El carácter de nuestra investigación en el que se emplea la experimentación y la observación nos permitió llegar a la verificación de nuestra hipótesis de que la canción es una herramienta efectiva en la enseñanza aprendizaje de la lengua francesa, especialmente, para mejorar las habilidades de la comprensión oral.

La canción "C'est de l'eau” fue una de las canciones más disfrutadas por parte de los estudiantes, tanto por el tema interesante como por sus intérpretes que se acercan a su edad. Además, el vídeo contiene imágenes parecidas a las que componen su vida diaria en el colegio, de modo que aprendieron una cantidad considerable de vocabulario relacionado al tema, y surgieron preguntas sobre el significado de algunas actividades relacionadas con el agua que no están en los ejercicios llevados a cabo. Esta sesión fue un ejemplo ideal porque la canción era adecuada para su edad y el tema era de su interés, algo que cumple el objetivo de esta investigación demostrando cómo se puede aprender un idioma extranjero disfrutando gracias a unas canciones que reflejan la vida real y no con los métodos más clásicos considerados aburridos por algunos estudiantes. Así utilizamos la canción, más allá del aprendiza- 
je del uso lingüístico para sensibilizar el alumnado sobre temas solidarios, humanistas, ciudadanos y universales, al mismo tiempo que enriquecíamos su vocabulario y su comprensión auditiva.

Por último, este trabajo puede servir como un punto de partida para otras investigaciones contando con una aplicación del experimento a mayor escala y con diferentes tipos de colegios. Además, se puede aplicar tanto en otros idiomas como en otros ámbitos educativos.

\section{Referencias bibliográficas}

\section{Libros}

Calvet, L.J., (1980) La chanson dans la classe de français langue étrangère. París, CLE International, Collection Outils théoriques.

Cohen, J., (1988) Statistical power analysis for the behavioral sciences. Denmark, Hillsdale, N.J, L. Erlbaum Associates.

Dumont, P., (1998) Le français par la chanson : nouvelles approches de l'enseignement de la langue et de la civilisation françaises à travers la chanson populaire contemporaine. París, L'Harmattan.

Mauger, G., (1955) Cours de Langue et de Civilisation Françaises. París, Hachette.

\section{Referencias bibliográficas digitales}

Boiron, M., (1997) Approches pédagogiques de la chanson [En línea]. Vichy, CAVILAM. Disponible en: <http://www.tv5monde.com/TV5Site/upload_image/app_ens/ens_ doc/26_fichier_approchechansons.pdf> [Último acceso el 21 de febrero de 2015].

Consejo de Europa, (2001) Marco común europeo de referencia para las lenguas: aprendizaje, enseñanza, evaluación [En línea]. Madrid, Ministerio de Educación, Cultura y Deporte. Disponible en: <http://cvc.cervantes.es/obref/marco $>$ [Último acceso el 25 de febrero de 2016].

Guan, X.. (2005) L'entraînement à l'utilisation des stratégies d'écoute - vers un enseignement plus efficace de la compréhension orale en L2 [En línea]. Lyon, Université de Lumière. Disponible en: $<\mathrm{http} / /$ theses.univ-lyon2.fr/documents/lyon2/2005/guan_x\#p=0\&a=top $>$ [Último acceso el 11 de febrero de 2016].

Morales, P., (2008) Estadística aplicada a las Ciencias Sociales [En línea]. Madrid, Universidad Pontificia Comillas. Disponible en: <https://www.academia.edu/9986406/estadistica_y_muestreo> [Último acceso el 20 de diciembre de 2015].

Morales, P., (2012) Análisis estadísticos combinando EXCEL y programas [En línea]. Madrid, Universidad Pontificia Comillas. Disponible en: <https://www.academia.edu/9002133/ An\%C3\%A1lisis_estad\%C3\%ADsticos_combinando_EXCEL_y_programas_de_Internet $>$ [Último acceso el 11 de noviembre de 2015].

Morales, P., (2013) Investigación experimental, diseños y contraste de medias [En línea]. Madrid, UNIVERSIDAD PONTIFICIA COMILLAS. Disponible en: $<$ https://www.academia.edu/6859778/Investigaci\%C3\%B3n_experimental_Investigaci\%C3\%B3n_experimental_dise $\% \mathrm{C} 3 \% \mathrm{~B} 10$ s_y_contraste_de_medias $>$ [Último acceso el 15 de diciembre de 2015].

Valentine, J. \& H. Cooper, (2003) Effect size substantive interpretation guidelines: Issues in the interpretation of effect sizes [En línea]. Washington, DC, Duke University. Disponible 
en: $<$ https:/www.wmich.edu/sites/default/files/attachments/u58/2015/Effect Size Substantive_Interpretation_Guideline.pdf $>$ [Último acceso el 19 de enero de 2016].

\section{Artículos de revistas:}

Boiron, M., (1998) "Rencontrer la musique française d'aujourd'hui", in Le Français dans le Monde. $\mathrm{N}^{\circ} 300$, pp. 36-39.

Boiron, M., (2001) "Chansons en classe, mode d'emploi", in Le Français dans le Monde. $\mathrm{N}^{\circ} 318$, p. 55.

Boiron, M. \& P. Hourbette, (1993) "La nouvelle génération française. De twist en rap: histoire", in Le Français dans le Monde. N257, pp. 52-56.

Calvet, L-J., (1977) “Alors, la chanson française?”, in Le Français dans le Monde. №131, p. 8 .

\section{Sitios web:}

Instituto Cervantes. CVC. Diccionario de términos clave de ELE. Comprensión auditiva [En línea]. Disponible en: <http://cvc.cervantes.es/ensenanza/biblioteca_ele/diccio_ele/ diccionario/comprensionauditiva.htm> [Último acceso el 10 de enero de $\overline{2016}$ ].

Les Enfantastiques. C'est de l'eau [En línea]. Disponible en: < https://www.youtube.com/ watch? $\mathrm{v}=\mathrm{jBFzQQ0C4CI}>$ [Último acceso el 10 de diciembre de 2015].

The Essential Guide to Effect Sizes. Effect size index [En línea]. Disponible en: < http://effectsizefaq.com/category/effect-size/ > [Último acceso el 10 de octubre de 2015].

TV5Monde. Canciones francófonas [En línea]. Disponible en: $<$ http://enseigner.tv5monde. com/collection/paroles-de-clips > [Último acceso el 25 de octubre de 2015]. 